\title{
Hypoplasia of lung a rare entity mimicking as a case of tuberculosis
}

\author{
Singh $\mathbf{U}^{1}$, Mohan $\mathbf{V}^{2}$, Goyal D.K ${ }^{3}$, Ramaraj $\mathbf{M}^{4}$, Shailly ${ }^{5}$, Poduvattil $\mathbf{P}^{6}$, Kaur R.P ${ }^{7}$, Abraham $\mathbf{J}^{8}$ \\ ${ }^{1}$ Dr. Urvinderpal Singh, Professor cum HOD, ${ }^{2}$ Dr. Vinay Mohan, Senior Resident, ${ }^{3}$ Dr. Deepak Kumar Goyal, Senior \\ Resident, ${ }^{4}$ Dr. Muralidharan Ramaraj, Junior Resident, ${ }^{5}$ Dr. Shailly, Junior Resident, ${ }^{6}$ Dr. Prasanth Poduvattil, Junior \\ Resident, ${ }^{7}$ Dr. Ritu Puneet Kaur, Junior Resident, ${ }^{8}$ Dr. Jebin Abraham, Junior Resident, all authors are affiliated with \\ Department of Tuberculosis and Chest diseases, Government Medical College, Patiala, Punjab, India.
}

Address for Correspondence: Dr. Vinay Mohan, Senior Resident, D/O Tuberculosis and Chest diseases, Government Medical College, Patiala, Punjab. Email id: vnybhagat@yahoo.co.in

\begin{abstract}
Pulmonary hypoplasia, a congenital malformation, is characterized by incomplete development of the lungs, leading to an abnormally less number or dimensions of bronchopulmonary segments / alveoli resulting in small fibrotic and nonfunctional lung. It is usually diagnosed in the neonatal period or in early childhood. Hypoplasia may be primary (idiopathic) or secondary. Primary pulmonary hypoplasia, not related to other congenital anomalies is extremely seldom diagnosed in adults.

We report the case of a 32-year-old married female with two kids presented to us with chief complaints of cough and fever for past nine months. Chest radiograph showed opacification of the left hemi-thorax with decrease in its size and marked ipsilateral mediastinal displacement with an increase in volume of right lung. After investigations she was diagnosed as a case of left lung primary pulmonary hypoplasia. High level of excellent clinical judgement needed to spot and diagnose this congenital aberration, often wrongly diagnosed in adults. Hence, once confronted with an opaque hemi-thorax in a young person with ipsilateral shift of the mediastinum, differential diagnosis of "lung hypoplasia" ought to be kept in mind.
\end{abstract}

Key-words: Congenital, Defects/Diseases, Hypoplasia, Pulmonary, Tuberculosis

\section{Introduction}

Congenital abnormalities of the lungs are very rare, and usually diagnosed prenatally or immediately after birth but extremely rare to be diagnosed in adults. Among the various inherent anomalies of the lung, pulmonary hypoplasia is a bronchopulmonary foregut anomaly associated with a numerical decrease and/or dimensions of airway generations, vessels and alveoli resulting in a small fibrotic, non -functioning lung [1]. The incidence of unilateral pulmonary agenesis is estimated to be between $0.0034 \%$ and $0.0097 \%$ [2]. Hypoplasia of the lung may be primary (idiopathic) un- associated with other anomalies [3] whereas secondary pulmonary hypoplasia is associated with other foetal developmental abnormalities like diaphragmatic congenital hernia, neuromuscular diseases, congenital heart diseases, thoracic cage anomalies (e.g. Jeune syndrome, asphyxiating thoracic dystrophy), urinogenitory tract abnormalities (e.g.Potter syndrome), chromosomal divergence, and pulmonary veno-lobar congenital syndrome (Scimitar syndrome). These disorders occur in neonates and have an adverse impact on the morbidity and survival [4].

\section{Case History}

A 32-year-old female presented to us with chief complaints of off and on cough with scanty expectoration for 9 months, off and on low grade fever with no diurnal variation and sore throat since 15 days. The cough was insidious in onset, dry

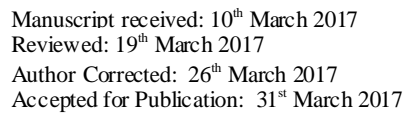


hacking in nature accompanied with scanty mucoid expectoration. There was no history of haemoptysis or chest pain. There was no history of breathlessness. In the family history, the patient is married and having two children. She was moderately built and nourished. Clinical examination of the chest showed that there was retracted left hemi-thorax with diminished movements. Trail sign was positive revealing marked shifting of trachea on the left side. Apex beat was felt in the mid axillary line on the left side. On percussion, resonant note was present in right hemi thorax and left supramammary and supra-scapular regions while dull note was present in left mammary, infra-mammary, axillary, infraaxillary, inter-scapular and infra-scapular regions. On auscultation, normal vesicular breath sounds were present in right hemi-thorax. Broncho-vesicular breath sounds were present in left supra-clavicular, infra-clavicular and supra-scapular regions. Breath sounds were absent in left mammary, infra-mammary, axillary and infra-scapular regions.

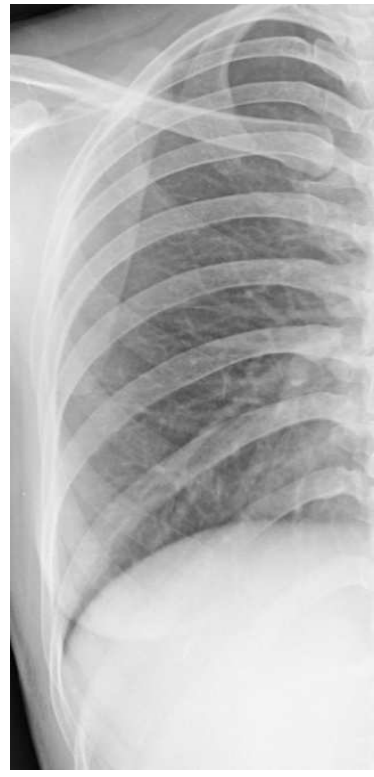

Figure 1: Postero-anterior chest radiograph showing opacification of the left hemithorax and shift of the mediastinum to the left.

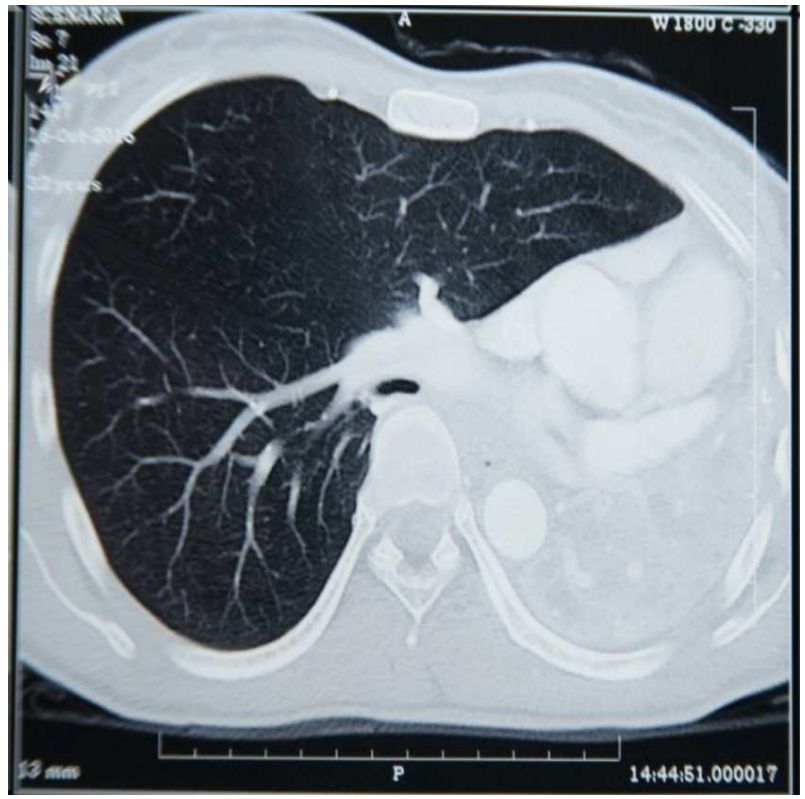

Figure 3: CECT of the chest showing hypoplastic left lung and displacement of the mediastinum to the left side.

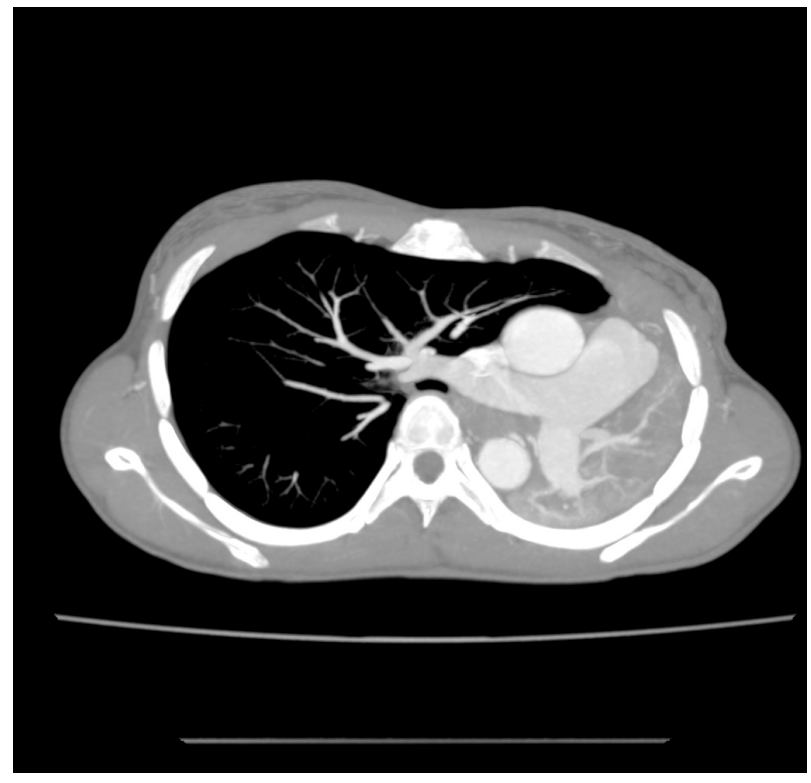

Figure 2: CECT of the chest showing hypoplastic left lung and displacement of the mediastinum to the left side.

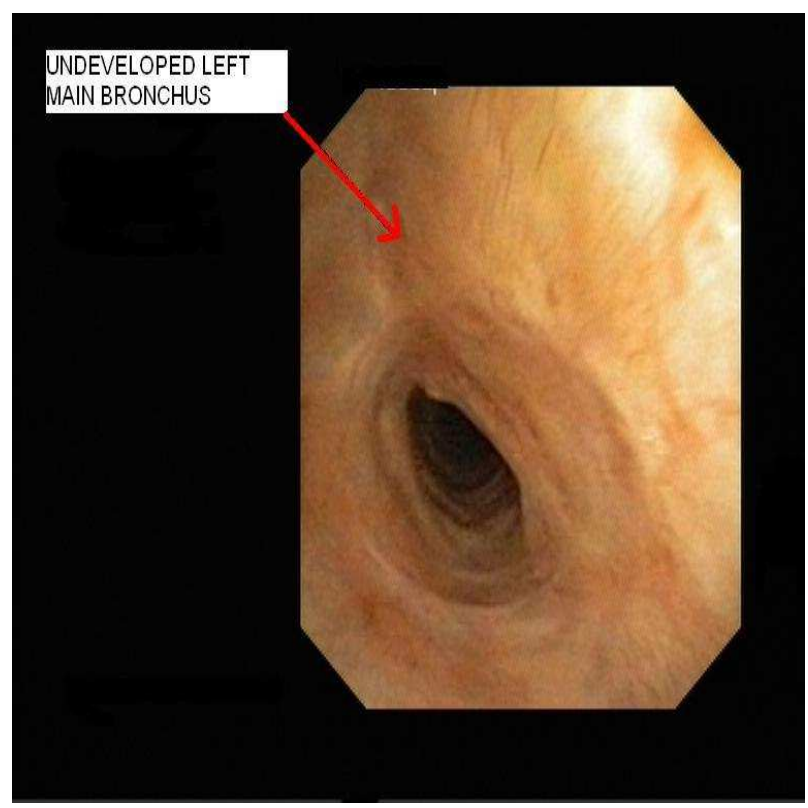

Figure 4: Bronchoscopic view at the carina showing the undeveloped left main bronchus. 


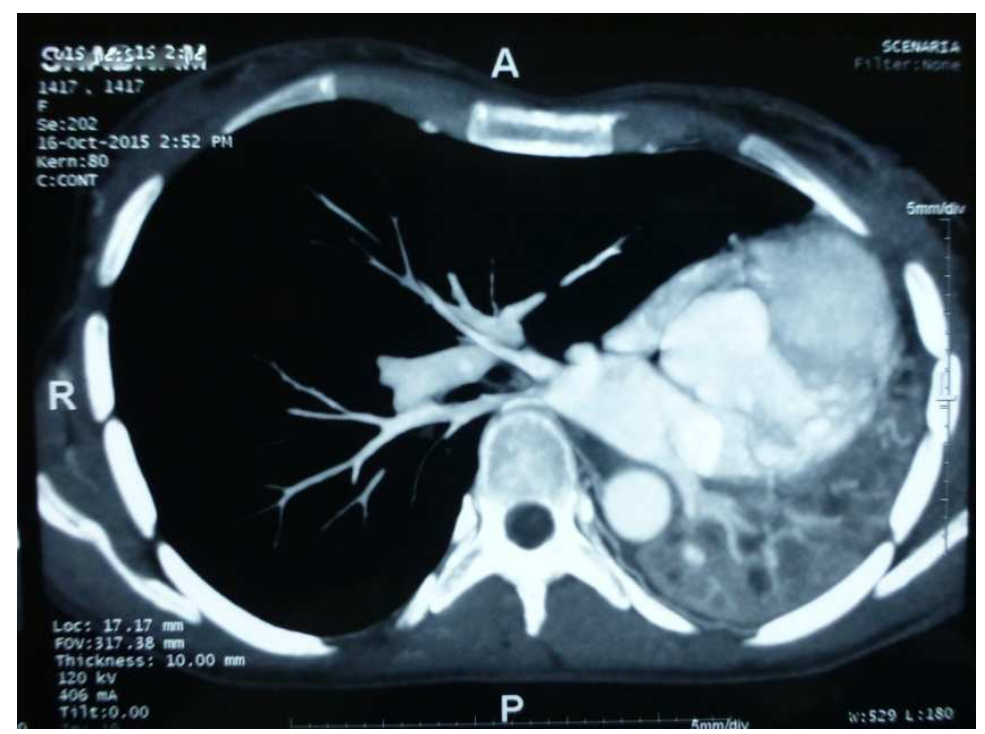

Figure 5: CT angiography showing a normal main pulmonary artery and both left and right pulmonary arteries.

All haematological and biological tests were normal except haemoglobin of 9.5 gm\%. Sputum for Acid Fast Bacilli (AFB) was negative for 2 consecutive days. Chest radiograph showed left hemi-thorax opacification with decrease in its size and marked ipsilateral mediastinal displacement with an increase in volume of right lung (Fig.1). Contrast enhanced computed tomography (CECT) of the chest demonstrated a hypoplastic left lung with ipsilateral displacement of the mediastinum. The right lung was hyper inflated with herniation to the contralateral side (Fig. 2,3). Fibroptic bronchoscopy revealed an undeveloped left main bronchus ending in a blind pouch just below the carina (Fig. 4). CE pulmonary angiography was done which revealed a normal main pulmonary artery and left and right pulmonary artery (Fig.5). Echocardiography, ultrasound of the abdomen and X-ray of the vertebral spine, which were carried out to ascertain any other associated congenital anomaly were normal.

Upon review of the history, examination and investigations diagnoses of Primary hypoplasia of the left lung was made. She was being treated for the upper respiratory tract infection (URI) including anti tubercular treatment without any definite evidence by the practitioners on the basis of lesions in Chest X-ray, which brought her to us for confirmation, and eventually led to the diagnosis of Hypoplasia of the left lung. The patient has been explained regarding her congenital anomaly with a further advice to report at this hospital immediately if she develops any symptoms related to the respiratory system.

\section{Discussion}

The development of the lungs and developmental malformations too takes place in the 3 rd and 4 th week of intrauterine life. Initially classified by Schneider and Schawatbe [5] and later modified by Boyden [6] depending upon the stage of development of the primitive bud, agenesis of the lung is further classified into 3 types.

Agenesis: (Type 1) is complete absence of the pulmonary artery and absence ipsilateral of pulmonary parenchyma and bronchus

Aplasia: (Type 2) is rudimentary bronchus with complete absence of pulmonary parenchyma.
Hypoplasia: (Type 3) is presence of variable amounts of pulmonary parenchyma, bronchi, and supporting vessels.

Our patient was type $\mathbf{3}$ as per this classification. Pulmonary hypoplasia either unilateral or bilateral, a condition of under development of the lung is due to a decrease in the number of the lung cells, alveoli and airways with a resulting decrease in the size and weight of the organ [7-9]. Hypo-plastic region of the lung becomes small, fibrotic and un-functional as a consequence of developmental delay in the alveolar tissue. Associated anomalies may be seen in several systems, especially in the urinary and musculoskeletal systems, cardiovascular and gastrointestinal [10]. 
Unilateral Primary ling hypoplasia is normally encountered in a child presenting with life- threatening symptoms (e.g. hypoxia, hypercapnia, tachypnea, cyanosis, early onset respiratory distress after birth and acidosis). However, it may be infrequently present in adults not producing any symptoms thus rendering its diagnosis problematic [11].

The chest X-ray shows hemi-thorax opacification with ipsilateral shift of the mediastinum. The markedly reduced volume is indicated by approximation of ribs, raised ipsilateral diaphagram, and shift of the mediastinum. Contralateral lung is greatly over inflated and there is herniation of the normal hyper inflated lung to the affected side. All these findings on X-ray were appreciated in our case also.

Contrast enhanced computed tomography (CECT) of the chest that provides a detailed and accurate lung parenchymal anatomy, bronchial tree and pulmonary vasculature, suffices to establish the extent of under development and to differentiate other radio-graphically mimicking conditions from hypoplasia. The other differentials of hemi-thorax opacification with mediastinum shift on the affected side like Collapse of the lung, Agenesis of the lung and fibrosis of the lung.

Although CECT is sufficient for the diagnosis of hypoplasia of the lung, but we carried out CT Angiography of the lung vasculature to visualize the pulmonary vessels and to rule out any vascular anomaly associated with Hypoplasia of the lung, such as scimitar syndrome as reported by Morgan JR et al [12].

Bronchoscopy may be carried out to visually examine the bronchial tree and to see the undeveloped/obliterated bronchus on the affected side as in our case. Hypoplasia of the lung is usually diagnosed antenatal or early after birth. However, it seems that patients with asymptomatic unilateral primary hypoplasia in adulthood exhibit increased life span due to compensatory contralateral lung hypertrophy that occupies the ipsilateral hemi-thorax.

The most favourable survival has been observed in cases of left lung hypoplasia because of the satisfactory compensatory hypertrophy of the larger right lung $[13,14]$, as also appreciated in our case.

Another point to mention is that our patient is married with two children and this anomaly was not noticed/ diagnosed during the delivery of two children further stressing the fact of favourable survival in cases of left lung hypoplasia without any other associated congenital anomalies.

Regarding management of primary pulmonary hypoplasia diagnosed in adults, asymptomatic cases need no intervention, but it is of paramount importance to prevent infection of the solitary lung. Infections of the solitary lung can be life-threatening and should be treated promptly [15]. Because of one functioning lung, these cases carry high-risk in any surgery. Asymptomatic cases and patients with minimal symptoms have good prognosis.

\section{Conclusion}

Unilateral hypoplasia of the lung, without any other congenital anomaly, is rarely seen. The disease is usually established in the perinatal or neonatal period. In adults, it requires a high level of good clinical acumen to identify and diagnose this congenital aberration. Thus, when confronted with an opaque hemi-thorax with shift of the mediastinum to the affected side in a young person, "hypoplasia of the lung" should be kept as one of the various differentials.

\section{Funding: Nil, Conflict of interest: None Permission of IRB: Yes}

\section{References}

1. Sundararajaperumal A, Vinodkumar V, Sundar V, Ranganathan D. Primary Pulmonary Hypoplasia in an adult. Pulmon 2008; 10(1):12-15.

2. Mardini MK, Nyhan WL. Agenesis of the lung. Report of four patients with unusual anomalies. Chest. 1985 Apr;87(4):522-7.

3. Devi YG, Rani NU, Rao GS, Narayana M, Kumar BD. A Rare Clinical Presentation of Primary Pulmonary Hypoplasia with Tuberculous Pleural Effusion. IJSS. 2014 Aug;1(3):16.

4. Currarino G, Williams B. Causes of congenital unilateral pulmonary hypoplasia: a study of 33 cases. Pediatr Radiol. 1985;15(1):15-24.

5. Schneider P, Schawatbe E. Die Morphologie der Missbildungen Des Menschen Under Thiere. 3 Part.2. Jena: Gustav Fischar.; 1912. pp. 817-22. [in German]. 
6. Cooney TP, Thurlbeck WM. Pulmonary hypoplasia in Down's syndrome. New England Journal of Medicine. 1982 Nov 4;307(19):1170-3.

7. Thurlbeck WM. Prematurity and the developing lung. Clinics in perinatology. 1992 Sep;19(3):497.

8. Lauria MR, Gonik B, Romero R. Pulmonary hypoplasia: pathogenesis, diagnosis, and antenatal prediction. Obstetrics \& Gynecology. 1995 Sep 1;86(3): 466-75.

9. Greenough A. Pulmonary Hypoplasia. In: Chervenak FA, Isaacson GC, Campbell S. Ultrasound in obstetrics and gynaecology. Boston: Little, Brown and company, corp, 1993: 903-8.

10. Cooney TP, Thurlbeck WM. and hydranencephaly. Cooney TP, Thurlbeck WM.Am Rev Respir Dis. 1985 Sep;132(3):596-601.

11. Pathania M, Lali BS, Rathaur VK. Unilateral pulmonary hypoplasia: a rare clinical presentation. BMJ
Case Rep. 2013 Mar 20;2013. pii: bcr2012008098. doi: 10. 1136/bcr-2012-008098.

12. Morgan JR, Forker AD. Syndrome of hypoplasia of the right lung and dextroposition of the heart:" scimitar sign" with normal pulmonary venous drainage. Circulation. 1971 Jan 1;43(1):27-30.

13. Berrocal T, Madrid C, Novo S, Gutiérrez J, Arjonilla A, Gómez-León N. Congenital Anomalies of the Tracheobronchial Tree, Lung, and Mediastinum: Embryology, Radiology, and Pathology 1. Radiographics. 2004 Jan;24(1):e17-.

14. Georgescu A, Nuta C, Bondari S. 3D Imaging in Unilateral Primary Pulmonary Hypoplasia in an Adult: A Case Report. Case Rep Radiol. 2011;2011:659586. doi: 10.1155/2011/659586. Epub 2011 Oct 19.

15. Singh U, Jhim D, Kumar S, Mittal V, Singh N, Gour H, Ramaraj M. Unilateral agenesis of the lung: a rare entity. Am J Case Rep. 2015 Feb 8;16:69.

\section{How to cite this article?}

Singh U, Mohan V, Goyal D.K, Ramaraj M, Shailly, Poduvattil P, Kaur R.P, Abraham J. Hypoplasia of lung a rare entity mimicking as a case of tuberculosis. Int J Med Res Rev 2017;5(03):315-319 doi:10.17511/ijmrr. 2017.i03.16. 